\title{
Acesso e Adesão ao Tratamento Oncológico Infantojuvenil: para além do Aspecto Médico-Biológico
}

doi: https://doi.org/10.32635/2176-9745.RBC.2018v64n3.48

Access and Adhesion to Oncological Treatment of Children and Adolescents: in Addition to the Medical-Biological Aspect

Acceso y Adhesión al Tratamiento Oncológico Infantojuvenil: además del Aspecto Médico-Biológico

Senir Santos da Hora ${ }^{1}$; Márcia Valéria de Carvalho Monteiro²; Simone Monteiro Dias ${ }^{3}$; Fernanda Ferreira da Silva Lima ${ }^{4}$; Josiane de Andrade Silva $^{5}$

\section{INTRODUÇÃO}

O câncer na faixa etária pediátrica é considerado uma doença rara. Apenas 3\% do total de casos de neoplasias malignas acometem crianças e adolescentes, de 0 a 19 anos de idade ${ }^{1}$. Contudo, destaca-se como a mais importante causa de óbito nessa população. Por não ter evidências confirmadas de que os fatores ambientais possam explicar o aparecimento do câncer infantojuvenil, não existem estratégias de prevenção primárias. Sendo assim, a maneira mais eficaz de controlar a doença em crianças e adolescentes é o diagnóstico precoce associado à garantia de um tratamento adequado.

O diagnóstico tardio do câncer infantojuvenil, as dificuldades para o acesso aos centros de tratamento e o abandono do tratamento podem acarretar inúmeras consequências desfavoráveis para as pessoas que vivenciam a doença. Entre essas consequências, estão as altas taxas de doença avançada, progressão da doença, necessidade de tratamentos mais intensos, maiores níveis de toxicidade, levando a piores prognósticos e resultados. Assim, a eficiência da rede pública de saúde é fundamental para identificação de sinais e sintomas importantes para o diagnóstico precoce e para o tratamento efetivo da doença ${ }^{2-4}$.

Diante dessa problemática, que não se restringe ao público infantojuvenil, estratégias têm sido adotadas no intuito de aumentar as chances de cura de pacientes com câncer. Para o início do tratamento, a Lei 12.732 de $22 / 11 / 2012$, em seu artigo $2^{\circ}$, prevê o prazo de até 60 dias contados a partir da data do resultado do laudo patológico ou em prazo menor ${ }^{5}$. Nesse cenário, é importante destacar que, de um modo geral, os cânceres pediátricos são frequentemente mais agressivos e rapidamente progressivos do que a maioria dos cânceres de adultos, o que torna premente o início imediato do tratamento.

O presente artigo busca identificar a influência das condições socioeconômicas, culturais e emocionais no acesso e na continuidade do tratamento oncológico infantojuvenil. Isto é, sinaliza-se o quanto os baixos níveis socioeconômicos associados aos aspectos subjetivos podem impactar no prognóstico de crianças e adolescentes com câncer, interferindo nas taxas de sobrevida, e, consequentemente, podendo contribuir no aumento significativo da mortalidade.

\section{DESAFIOS À GARANTIA DO ACESSO E ADESÃO AO TRATAMENTO ONCOLÓGICO INFANTOJUVENIL}

A sobrevida de crianças e adolescentes com câncer vem apresentando melhora, nos últimos anos, principalmente nos países economicamente desenvolvidos, em que as taxas de sobrevida em cinco anos já chegam a $80 \%{ }^{6}$. Nos países periféricos, como o Brasil, apesar dos avanços no diagnóstico e tratamento, as taxas de sobrevida ainda não alcançaram essa paridade. A estimativa por câncer infantojuvenil no país, por meio do índice calculado com base nas informaçóes de incidência e mortalidade divulgadas pelo Instituto Nacional de Câncer José Alencar Gomes da Silva (INCA) e pelo Ministério da Saúde em 2016, é de 64\%, uma taxa ainda baixa em relação aos países desenvolvidos?

A Organização Mundial da Saúde (OMS) sinaliza que, a cada ano, mais de 150 mil crianças são diagnosticadas com câncer em todas as regióes do mundo. A organização ainda informou, em nota, que, por meio do acesso a serviços de saúde de qualidade, mais de $80 \%$ das crianças com câncer podem sobreviver, com direito a uma vida

\footnotetext{
1 Instituto Nacional de Câncer José Alencar Gomes da Silva (INCA). Orcid iD: https://orcid.org/0000-0002-0161-3701.

${ }^{2}$ INCA. Orcid iD: https://orcid.org/0000-0002-3264-2994

${ }^{3}$ INCA. Orcid iD: https://orcid.org/0000-0003-3390-0964

${ }^{4}$ INCA. Orcid iD: https://orcid.org/0000-0002-6658-3101

${ }^{5}$ Centro Universitário Augusto Motta (Unisuam). Orcid iD: https://orcid.org/0000-0002-1207-7780

Endereço para correspondência: Senir Santos da Hora. Travessa Durval Corrêa de Sá, 26 - Serra do Sambe. Rio Bonito (RJ), Brasil. CEP $24800-000$.

E-mail: senirsantos@hotmail.com.
} 
plena e saudável ${ }^{8}$. Entretanto, muitas crianças em países de baixa e média rendas não recebem tratamento completo e, como resultado, mais de $80 \%$ das mortes por câncer infantil são registradas em localidades com pouco recurso?

O diagnóstico tardio e o tratamento inacessível do câncer vêm se tornando comuns nos países de baixa renda ${ }^{8}$. Em 2017, apenas 26\% dos países de baixa renda sinalizaram a existência de serviços de patologia disponíveis no setor público. Enquanto mais de $90 \%$ dos países de alta renda relataram a eficiência dos serviços de tratamento. Consequentemente, os dados apontam que, a partir de 2012, o câncer foi a segunda principal causa de morte no mundo, com 8,2 milhóes de mortes; a maioria ocorreu em países de baixa e média rendas ${ }^{8}$.

Buscando enfrentar essa disparidade entre os países e reduzir a mortalidade prematura por câncer, em maio de 2017, durante a 70a Assembleia Mundial da Saúde (realizada em Genebra, Suíça), foi aprovada uma resolução de prevenção e controle do câncer por meio de uma abordagem integrada ${ }^{8}$. Ao considerar que certos grupos populacionais experimentam desigualdades na exposição a fatores de acesso à triagem, ao diagnóstico precoce e ao tratamento oportuno e adequado; e que diferentes estratégias de controle do câncer são necessárias para grupos específicos de pacientes com câncer, como crianças e adolescentes, a resoluçáo possibilitou discutir algumas estratégias para o diagnóstico precoce e o tratamento rápido e adequado, incluindo alívio da dor e cuidados paliativos.

No escopo de reduzir a mortalidade e melhorar os resultados e a qualidade de vida dos pacientes com câncer, algumas pactuaçôes foram possíveis, entre elas, destacam-se: a implementação de intervençóes de prevenção, detecção precoce, rastreamento, tratamento e cuidados paliativos e de sobrevivência, inclusive para câncer na infância.

Considerando-se que as condiçóes socioeconômicas associadas aos fatores emocionais e culturais têm interferência significativa no processo de acesso e adesão ao tratamento e, portanto, de recuperaçáo da doença, torna-se fundamental avaliar o universo cultural e emocional, os recursos socioeconômicos das famílias de crianças e adolescentes com câncer. Nesse entendimento, os obstáculos à continuidade do tratamento se colocam como foco de atençáo da equipe de saúde multiprofissional, de modo a garantir o direito desse público à saúde e à vida, conforme preconiza o Estatuto da Criança e do Adolescente $(\text { Ecriad })^{10}$.

\section{DEMANDAS NO TRATAMENTO ONCOLÓGICO INFANTOJUVENIL: ALÉM DO ASPECTO MÉDICO-BIOLÓGICO}

O câncer ainda é uma doença estigmatizada, associada à dor, ao sofrimento e à morte. Em relação à infância, o adoecer e a proximidade com o morrer nessa fase da vida são entendidos como uma ruptura com o ciclo natural e causam intenso sofrimento psíquico entre os membros da família.

Ao impacto emocional, somam-se profundas alteraçóes nas relaçốes familiares e na vida das crianças e adolescentes expostos a repetidos procedimentos médicos invasivos, dos quais decorrem efeitos colaterais, dor, mudanças na autoimagem e incertezas sobre o tratamento. Assim, o câncer afeta não só a dimensão biológica, mas também outras dimensóes, como a interrupção da rotina social e escolar, das atividades de lazer, com a suspensão do convívio com outras crianças e com os membros da família. Tudo isso em um contexto socioeconômico nem sempre favorável ao grupo familiar, muitas vezes, marcado por condiçôes adversas de existência e subsistência.

O processo de tratamento oncológico infantojuvenil impóe alteraçóes na rotina profissional da família, que necessita de adaptaçôes financeiras decorrentes do aumento dos gastos e da necessidade de acompanhamento contínuo. Por ser um tratamento longo e desgastante, muitas famílias apresentam dificuldades de conciliar as atividades do cuidado e do trabalho, contribuindo para que um dos pais ou responsáveis optem por abrir mão de seu emprego para estar ao lado de sua prole em tempo integral. Cabe ressaltar as famílias cujo cuidador é também o provedor, o que implicará na reduçáo de renda ao longo do tratamento e na maior pauperização dos seus membros, interferindo diretamente na qualidade de vida e na saúde.

Nas famílias em que os pais trabalham com vínculo empregatício, não há respaldo legal na Consolidação das Leis do Trabalho (CLT) para que estes se afastem do trabalho durante o acompanhamento de seus filhos. A Lei $13.257 / 2016^{11}$, que incluiu os incisos X e XI, no art. $473 \mathrm{da}$ CLT, assegura apenas o abono de um dia por ano, em virtude de atestado de acompanhamento médico para levar o filho de até 6 anos em consulta médica.

Observa-se um paradoxo, as crianças e os adolescentes têm o direito de acompanhamento no período de internaçáo, sendo dever da família, da sociedade e do Estado a garantia da vida e da saúde, como preconizados no Ecriad $^{10} \mathrm{em}$ seu art. $4^{\circ}$, mas a CLT não assegura a licença aos pais para tal acompanhamento, evidenciando uma explícita violação do direito da criança e do adolescente em tratamento de saúde, de ter a manutenção do acompanhamento por parte dos responsáveis.

Outro fator que também pode contribuir para aumentar a condição de vulnerabilidade é a situação habitacional no que se refere à infraestrutura básica, o que envolve questôes como as condiçôes da construção, as dimensões do imóvel, o abastecimento de água, o fornecimento de energia elétrica e o saneamento básico, nem sempre favoráveis à permanência da criança no domicílio; além das condições de acesso ao centro de 
tratamento, tais como disponibilidade de transporte coletivo urbano, rua íngreme, área rural ou urbana, o que dificulta o deslocamento diário. Há também questóes provenientes do universo cultural, entre as quais se destacam as famílias com baixa capacidade cognitiva, a despeito do grau de escolaridade, e as famílias cuja visão de mundo se paute apenas nos valores e crenças religiosas, que interferem no processo de tratamento, impondo desafios à equipe de saúde.

Considerando o conceito ampliado de saúde como resultado das condiçôes de vida da população, o trabalho com famílias de crianças e adolescentes em tratamento oncológico pressupóe integralidade das açôes, como interdisciplinaridade e intersetorialidade. A intersetorialidade requer a articulação entre os níveis de atenção em saúde e destes com as demais políticas públicas; enquanto a interdisciplinaridade exige troca de saberes e compartilhamento de decisôes e açôes entre os membros da equipe, o que contribui para a ampliação de espaços de partilha e interlocução entre família e equipe, estabelecendo nexos entre as condiçóes socioeconômicas, emocionais, culturais e a complexidade do tratamento oncológico.

\section{CONCLUSÃO}

Conclui-se, dessa forma, que a continuidade do tratamento está relacionada às questóes socioeconômicas, emocionais e culturais das crianças e adolescentes e de suas famílias. Embora, muitas vezes, elas possuam os melhores tratamentos em centros especializados com as mais avançadas tecnologias, nem sempre possuem meios de acessá-los.

A experiência aponta que tais condiçóes de acesso estáo relacionadas ao precário contexto socioeconômico das famílias e à ausência de suporte efetivo às demandas geradas durante o tratamento oncológico. As contribuiçôes institucionais, que seriam capazes de diminuir a condição de vulnerabilidade das famílias, estão associadas à integralidade das açóes entre os membros da equipe de saúde, na articulação e mobilização dos recursos e serviços da rede de proteção social e na viabilização de acesso aos direitos decorrentes da patologia e aos demais direitos sociais garantidos pelas políticas públicas.

\section{CONTRIBUIÇÕES}

Todas as autoras contribuíram em todas as etapas do manuscrito.

\section{DECLARAÇÃO DE CONFLITO DE INTERESSES}

Nada a declarar.

\section{FONTES DE FINANCIAMENTO}

Não há.

\section{REFERÊNCIAS}

1. Instituto Nacional de Câncer José Alencar Gomes da Silva. Estimativa 2018: incidência de câncer no Brasil. Rio de Janeiro: INCA; 2017.

2. Howard SC, Wilimas JA. Delays in diagnosis and treatment of childhood cancer: where in the world are they important? Pediatr Blood Cancer. 2005 Apr;44(4):303-4.

3. Mostert S, Arora RS, Arreola M, Bagai P, Friedrich P, Gupta $S$, et al. Abandonment of treatment for childhood Cancer: position statement of a SIOP PODC Working Group. Lancet Oncol. 2011 Aug;12(8):719-20.

4. Howard SC, Marinoni M, Castillo L, Bonilla M, Tognoni G, Luna-Fineman S, et al. Improving outcomes for children with cancer in low-income countries in Latin America: a report on the recent meetings of the Monza International School of Pediatric Hematology/ Oncology (MISPHO)-Part I. Pediatr Blood Cancer. 2007 Mar;48(3):364-9.

5. Presidência da República (BR). Lei $\mathrm{n}^{\circ}$ 12.732, de 22 de novembro de 2012 [Internet]. [acesso 2018 ago 29]. Disponível em: http://www.planalto.gov.br/ CCIVIL_03/_Ato2011-2014/2012/Lei/L12732.htm.

6. Smith MA, Seibel NL, Altekruse SF, Ries LA, Melbert DL, O'Leary M, Outcomes for children and adolescents with cancer: challenges for the twenty-first century. J Clin Oncol. 2010 May 20;28(15):2625-34.

7. Instituto Nacional de Câncer José Alencar Gomes da Silva. Incidência, mortalidade e morbidade hospitalar por câncer em crianças, adolescentes e adultos jovens no Brasil: informaçóes dos registros de câncer e do sistema de mortalidade. Rio de Janeiro: INCA; 2016.

8. World Health Organization. Cancer prevention and control in the context of an integrated approach: WHA Resolution; Seventieth World Health Assembly; WHA70.12 [Internet]. Geneve: WHO; 2017 [cited 2018 Ago 29]. Available from: http://apps.who.int/ medicinedocs/documents/s23233en/s23233en.pdf.

9. Rodriguez-Galindo C, Friedrich P, Alcasabas P, Antillon F, Banavali S, Castillo L, et al. Toward the cure of all children with cancer through collaborative efforts: pediatric oncology as a global challenge. J Clin Oncol. 2015 Sep 20;33(27): 3065-73.

10. Presidência da República (BR). Lei $n^{\circ} 8.069$, de 13 de julho de 1990 [Internet]. [acesso 2018 Ago 29]. 
Disponível em: http://www.planalto.gov.br/ccivil_03/ LEIS/L8069.htm.

11. Presidência da República (BR). Lei 13.257, de 08 de março de 2016 [Internet]. [acesso 2018 Ago 29]. Disponível em: http://www.planalto.gov.br/ccivil_03/_ ato2015-2018/2016/Lei/L13257.htm.

Recebido em 12/9/2018

Aprovado em 20/11/2018 